\title{
An Approach to Estimate Aircraft Fuel Consumption for the Descent Phase
}

\author{
Özlem Şahin ${ }^{1 *}$, Çerkez Ağayeva ${ }^{2}$ \\ ${ }^{1}$ Department of Air Traffic Control, Faculty of Aeronautics and Astronautics, Eskişehir Technical University, \\ Eskisehir, Turkey \\ ${ }^{2}$ Department of Economics, Economics and Administrative Sciences, Mus Alparslan University, Mus, Turkey \\ *ozlemsahin@eskisehir.edu.tr
}

Received: 13 March 2018

Accepted: 27 November 2018

DOI: $10.18466 /$ cbayarfbe. 405224

\begin{abstract}
In this study, the static (based on Base of Aircraft Data) and the dynamic aircraft fuel consumption models are constructed for the descent phase. Therefore, two flight trajectories, from Istanbul to Frankfurt (LTBAEDDF) and from Los Angeles to New York (KLAX-KJFK), are chosen in order to analyze the appropriate relationships between the different parameters such as altitude, speed and time. A medium range aircraft is taken into consideration while developing linear and quadratic models. For LTBA- EDDF, fuel consumption as $200 \mathrm{~kg}, 193,89 \mathrm{~kg}$ and $193,58 \mathrm{~kg}$ are found for BADA, linear and quadratic models, respectively. It corresponds to about a $3 \%$ difference from BADA. Furthermore, for KLAX-KJFK, approximately a 5\% difference is obtained.
\end{abstract}

Keywords: Aircraft, fuel consumption, descent phase, static and dynamic models.

\section{Introduction}

Fuel cost and environmental concern have been rising in aviation industry. Therefore, calculating the precise and exact aircraft fuel consumption gains an importance in order to reduce environmental impacts and also provide economic aircraft operations. Managing and handling air traffic flows in accurate methods and also the exact and optimal design of flight procedures are critical in order to provide efficiency in fuel consumption. For instance, one of the methods for reducing the fuel consumption is to design an optimal operational flight procedure such as optimized profile descent (OPD) sometimes referred to as continuous descent operations (CDO). Moreover, it could be noted that arrival routes based on area navigation (RNAV), such as a point merge system (PMS), will also reduce fuel consumption. Because these procedures allow aircraft descending from an optimal position with minimum engine thrust. Moreover, area navigation route structures generally enable aircraft to fly in direct paths.

Aviation industry (airlines, aircraft manufacturers, regulatory organization, etc.) is affected by the fuel consumption and its economic costs. In decades, several solutions have been researched so as to reduce the fuel consumption and minimize its economic influence to airliners. Furthermore, it will pioneer to reduce the greenhouse gas emissions and illnesses caused by fuel consumption [1]. Several kinds of fuel consumption models exist. In the following paragraphs, the studies related to fuel consumption models will be summarized.
Aviation stakeholders have worked to develop algorithms in order to model fuel consumption. In the early 1980s, Collins [2] worked on the more accurate fuel consumption model for improving the measurement of fuel efficiency for the airlines. In the recent years, Trani et al. [3] have researched the use of neural networks (NN) for estimating fuel consumption. Fokker F-100 aircraft performance data was used by Trani et. al. [3, 4]. Senzig et al. [1] marked that these models have acquired limited acceptance due to many of reasons such as the requirements of detailed airplane aerodynamic information and a large database of airplane operations. A thrust specific fuel consumption model which related to Mach number and excluded the effect of temperature ratio was developed by Bartel and Young. That model was improved for the cruise phase [5].

The existing fuel consumption models based on International Civil Aviation Organization (ICAO) reference emissions landing and takeoff (LTO) cycle method and Eurocontrol's base of aircraft data (BADA) have won wide use in environmental analyses. Patterson et al. [6] have indicated that the ICAO time-in-mode method is not prototype of real world operations. Energybalance thrust model is used by BADA fuel consumption model and Thrust Specific Fuel Consumption (TSFC) is as a function of airspeed. In the cruise phase, BADA fuel consumption model works well as detailed by Malwitz et al. [7] and Lee et al. [8]. However, in the terminal area, BADA is compared to airline fuel consumption which is obtained via flight data record (FDR) system and it is revealed that BADA does not achieve as accurately in this region. Senzig et al. noted that an improved and more accurate new fuel consumption model is needed for the 
terminal area. Hill and Petersen [9] proposed a departure TSFC model uses linear relationships for Mach number, thrust and altitude, which are functions of TSFC and it is presented in Eq. (1). K1, K2, K3, K4 are expressed the individual aircraft types.

$T S F C / \sqrt{\theta}=K_{1}+K_{2} M+K_{3} h_{M S L}+K_{4} F / \delta$

Hill and Petersen's proposed arrival TSFC model with modifications by Yoder has been simplified. The proposed arrival TSFC model is presented in Eq. (2) which indicates arrival fuel consumption in terminal area [1]

$$
T S F C / \sqrt{\theta}=\alpha+\beta_{1} M+\beta_{2} e^{-\beta_{3}\left(F / \delta / F_{0}\right)}
$$

where $\alpha$ and $\beta$ i are individual arrival coefficients.

Turgut and Rosen [10] investigated the relationship between fuel flow and altitude during descent phase for commercial aircraft using genetic algorithm (GA). They established exponential models between fuel flow and altitude. The results are compared with the actual flight data obtained from FDRs. The predictions of models show similar results to actual observations. Turgut and Rosen highlighted that keeping aircraft at a higher altitude as soon as possible achieves less fuel consumption during descent.

Numerous studies and approaches are available regarding the estimation of aircraft fuel consumptions i.e regression model of Stolzer [11], aero-propulsive model of Gong and Chan [12] based on flight manual data, physics-based model of Allaire [13], neural networks and GA model of Spencer [14], fuel burn models of Ryerson et al. [15] and Chati et al. [16] based on operation data.

In this study, an exponential fuel consumption model with respect to altitude for descent phase is developed. The Airbus 320 aircraft performance data obtained from BADA is used for calculating fuel consumption for real traffic. These real traffic data are provided from flight aware online website. On the other hand, dynamic change of the altitude respect to time in the descent phase is investigated and the proposed model is enhanced by using some statistical tools. The fuel consumption results are calculated in MATLAB and compared for two real flight routes.

The remainder of this article is organized as follows: section 2 gives a detailed account of the methodology. In section 3, the static and dynamic fuel consumption models are given and applied to real air traffic data. Finally, section 4 gives the conclusions.

\section{Materials and Methods}

It is well known that the fuel consumption models are closely dependent on a procedure of flights. Firstly, two real flight operations are introduced and analyzed for clarity and a better understanding.

The following two flight travels are considered:

- Istanbul-Frankfurt (LTBA-EDDF)

\section{- Los Angles-New York (KLAX-KJFK)}

The flight from Istanbul to Frankfurt is an international flight. The planned flight distance between two airports is 1160 nautical miles $(\mathrm{nm})$. The total time of flight, namely from takeoff to landing, is approximately 2,5 hours. The type of aircraft is a medium range aircraft Airbus 320. Aircraft reaches at 36,000 feet in the cruise phase.

Another flight from Los Angles to New York is a domestic flight approximately takes 5 hours. The planned flight distance is $2650 \mathrm{~nm}$. In this flight operation medium range aircraft $\mathrm{A} 320$ is used, too. The aircraft does not reach the maximum cruise altitude; it maintains 37,000 feet at cruise.

Aircraft trajectory data derived from "flight aware" [16] website is examined by planned flight distance, actual flight distance, flight duration and difference between planned and actual flight distance. The maximum (Dist_Max), minimum (Dist_Min) and average (Dist_Ave) variation, which are the values between planned and actual flight distances, are computed. The value of average flight distance is chosen to be analyzed in this study. Flight trajectories belonging to LTBAEDDF and KLAX-KJFK are presented in Figure 1 and Figure 2.

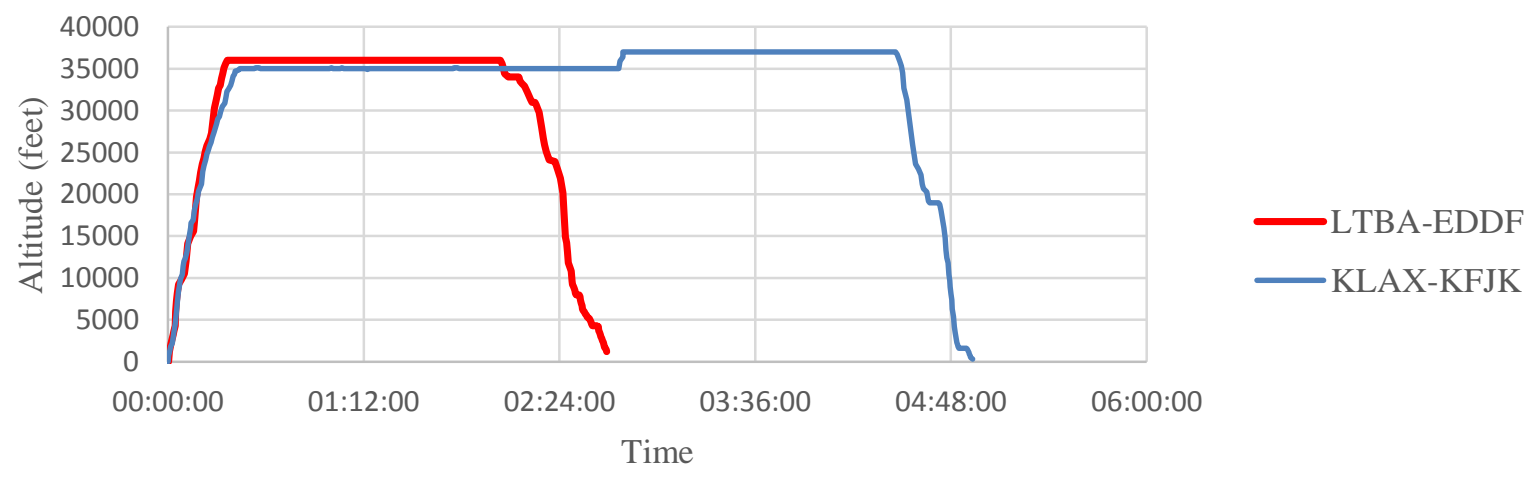

Figure 1. Flight trajectories for LTBA-EDDF and KLAX-KJFK in terms of altitude and time. 


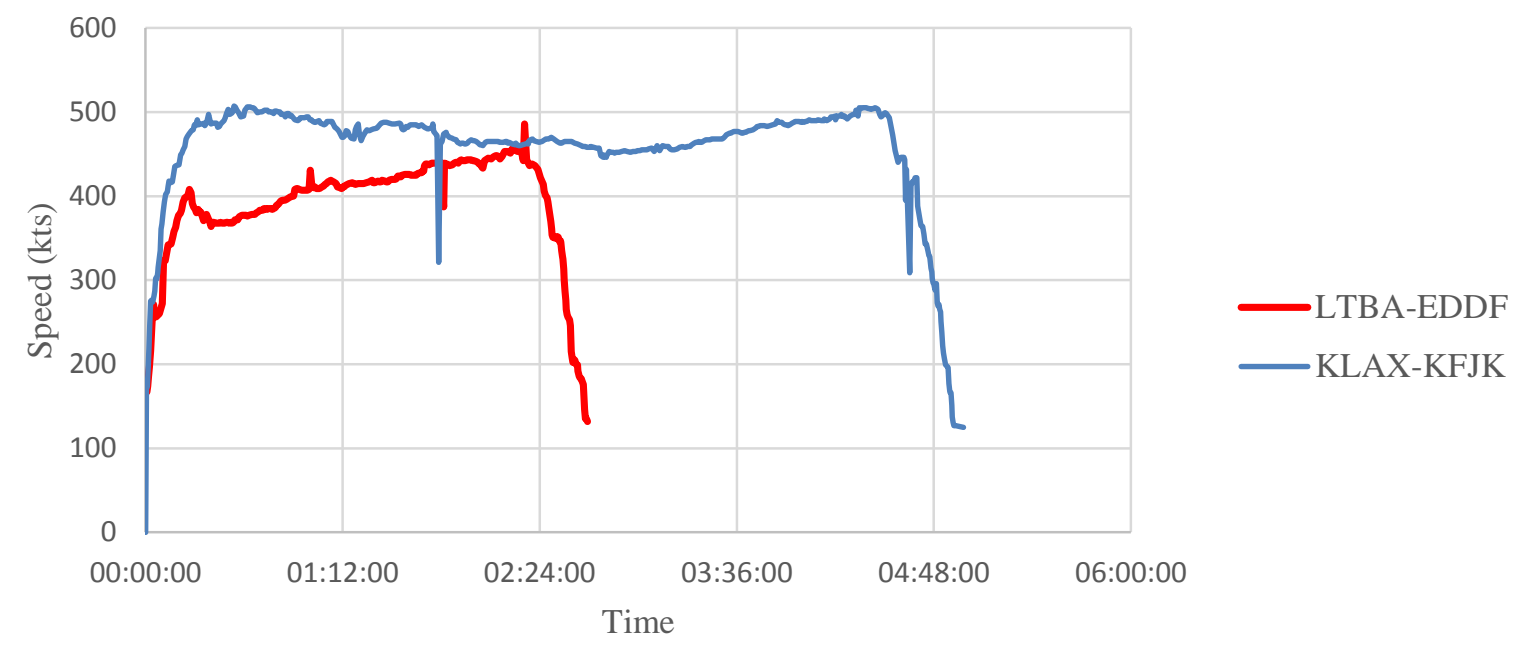

Figure 2. Flight trajectories for LTBA-EDDF and KLAX-KJFK in terms of speed and time.

According to the Dist_Max, Dist_Min and Dist_Ave, the flight duration for climb, cruise and descent phases are indicated in Table 1 and Table 2.

Table 1. The flight times in minutes according to phases for LTBA-EDDF.

\begin{tabular}{|l|c|c|c|c|}
\cline { 2 - 5 } \multicolumn{1}{c|}{} & \multicolumn{4}{c|}{ Time (minutes) } \\
\cline { 2 - 5 } \multicolumn{1}{c|}{} & Climb & Cruise & Descent & Total \\
\hline Dist_Max & 21 & 110 & 39 & 170 \\
\hline Dist_Ave & 21 & 101 & 40 & 162 \\
\hline Dist_Min & 25 & 91 & 32 & 148 \\
\hline
\end{tabular}

Table 2. The flight times in minutes according to phases for KLAX-KJFK.

\begin{tabular}{|l|c|c|c|c|}
\cline { 2 - 5 } \multicolumn{1}{c|}{} & \multicolumn{4}{c|}{ Time (minutes) } \\
\cline { 2 - 5 } \multicolumn{1}{c|}{} & Climb & Cruise & Descent & Total \\
\hline Dist_Max & 29 & 231 & 35 & 295 \\
\hline Dist_Ave & 26 & 241 & 28 & 295 \\
\hline Dist_Min & 33 & 219 & 28 & 280 \\
\hline
\end{tabular}

\subsection{A Proposed Fuel Consumption Model}

BADA (version 3.6) developed by Eurocontrol includes true airspeed, rate of descent, rate of climb and fuel flow rate in terms of flight levels at cruise, descent and climb phases of flights.

As mentioned before, while estimating the fuel consumption, it is unavoidable that some errors occur. Some views related to version of BADA are investigated and the results are remarked that the final approach speed of aircraft are extremely high and also unreliable thrust specific fuel consumption (TSFC) is obtained [8].

The main idea of fuel model in BADA is based on Thrust Energy Model (TEM) which is presented as follows:
$(T-D) V_{T A S}=m g \frac{\partial h}{\partial t}+m V_{T A S} \frac{\partial V_{T A S}}{\partial t}$

Here, "T" expresses Thrust, "D" denotes Drag, aircraft mass is presented as " $\mathrm{m}$ ", and geodetic altitude is indicated as "h", gravitational acceleration and true airspeed are represented as "g" and "V $\mathrm{VAS}_{\mathrm{AS}}$ ", respectively.

The above mentioned equation (3) shows that less thrust is needed in descend phase rather than in cruise phase. Because the acceleration $\left(\frac{\partial V_{T A S}}{\partial t}\right)$ on descent is negative, however, in cruise phase it is null.

The analyzing of fuel consumption is closely related with TSFC $(\eta)$ characteristics. The fuel consumption depends on several of parameters such as flap settings, air density, the wing reference area etc. Because of that reason general expression of TSFC is enough complex. Faced with difficulties in application of these formula, it requires a simplification of the corresponding expression. One of such approaches which gives more accurate values is developed by Senzig et al. below in Eq. 4

$\eta=\alpha+\beta M+\gamma e^{-\theta \frac{T}{F}}$

where " $\mathrm{M}$ " is the Mach number, " $\mathrm{F}$ " is the static thrust, $\alpha, \beta, \gamma, \theta$ are empirical coefficients.

In addition, the estimation of parameters such as $\mathrm{M}, \mathrm{T}, \mathrm{F}$ based on several kinds of atmospheric parameters such as, temperature, density, and engine types, speed, throttle settings, etc.

Going from the idea of exponential dependence in Eq. 4 and a proposed model which is a function of an altitude is given in Eq. 5. The proposed fuel consumption model is developed based on BADA and the model.

$f=\beta e^{-\propto h}$ 


\section{Results and Discussion}

The Airbus 320 is a twin-engine, medium range jetliner, widely used in the air transportation industry is chosen for this study. The flight trajectory data for the study are achieved by flight aware [17] website. The coefficients based to BADA aircraft performance in Eq. (5) are calculated:

$\beta=9,22 ; \quad \propto=1,659 \times 10^{-5}$

In this study, only the descent phase is taken into consideration and the fuel consumption is calculated using an exponential model based on BADA. Besides, real traffic data appear varying according to time; therefore, the dynamic change of altitude respect to time is investigated. In terms of the obtained model, fuel consumption is computed using MATLAB.

For two flight routes, LTBA-EDDF and KLAX-KJFK, the dynamic change of the altitude is examined respect to elapsed time from the start of flight, in the descent phase.

Dynamical changes in the route from LTBA to EDDF are acquired as follow in Eq. 6 and Eq. 7:

Linear dependence:

$h_{1}(t)=167286,7-1041,98 t, \mathrm{R}^{2}=0,963 ;$

Quadratic dependence:

$h_{2}(t)=132345,54-549,93 t-1,72 t^{2}, \mathrm{R}^{2}=0,963$

(7)

Using above mentioned expressions, we can achieve next models of fuel consumption in the descent phase for each case (Eq. 8 and Eq.9):

$$
\begin{aligned}
f_{1}(t) & =\beta e^{-\alpha(167286,7-1041,98 t)}, \\
f_{2}(t) & =\beta e^{-\alpha\left(132345,54-549,93 t-1,72 t^{2}\right)}
\end{aligned}
$$

Similar analysis for the route from KLAX to KJFK is defined that the linear dependence (Eq. 10):

$h_{1}(t)=407847,49-1385,86 t, \mathrm{R}^{2}=0,966 ;$

and the quadratic dependence (Eq. 11):

$$
\begin{aligned}
& h_{2}(t)=432532,9-1560,96 t+0,314 t^{2}, \\
& \mathrm{R}^{2}=0,966
\end{aligned}
$$

are the best dynamical models in the descent phase. Consequently, fuel consumption in the descent phase is defined in Eq. 12 and Eq. 13:

$f_{1}(t)=\beta e^{-\alpha(407847,49-1385,86 t)}$,

$f_{2}(t)=\beta e^{-\alpha\left(432532,9-1560,96 t+0,314 t^{2}\right)}$

for linear and quadratic dependence respectively.

As it is known that the phases of a flight are takeoff, climb, cruise, descent, approach and landing. Looking through BADA, we observed a significant variation in fuel consumption respect to an altitude. In this paper, altitude classification is proposed and is used due to the variation of fuel consumption observed among these altitudes. Table 3 represents the altitude classification and the fuel flow rate $(\mathrm{kg} / \mathrm{min})$.

The altitude ranges between $39,000 \mathrm{ft}$ and 3,000 ft is assumed to the descent phase. The approach phase is considered between 3,000 ft and 1,500 ft and after which the aircraft starts landing (Table 3). The altitude classification changes in terms of aircraft types.

Table 3. Altitude classification based on BADA.

\begin{tabular}{|c|c|c|c|c|c|c|}
\hline Aircraft type & \multicolumn{3}{|c|}{ Altitude (*100 ft) } & \multicolumn{2}{c|}{$\begin{array}{c}\text { Fuel consumption (kg/min) } \\
\text { (range between) }\end{array}$} \\
\hline \multirow{3}{*}{ A320 } & Descent & Approach & Landing & Descent & Approach & Landing \\
\cline { 2 - 7 } & $390-30$ & $30-15$ & $15-0$ & $4,7-8,6$ & $16,0-16,2$ & $42,7-43,5$ \\
\hline
\end{tabular}

In Table 4, two flight routes are analyzed and time intervals are obtained according to the altitude classification. Moreover, the fuel consumption $(\mathrm{kg})$ are calculated for linear and quadratic models. BADA results are compared with the proposed models and the results are found to be similar.

Table 4. Fuel consumption $(\mathrm{kg})$ for two flight routes.

\begin{tabular}{|l|c|c|c|c|c|}
\hline \multirow{4}{*}{ Flight routes } & \multicolumn{2}{|c|}{ Parameters } & \multicolumn{2}{c|}{ Fuel consumption (kg) } \\
\cline { 2 - 6 } & $\begin{array}{c}\text { Altitude } \\
(* \mathbf{1 0 0 f t})\end{array}$ & $\begin{array}{c}\text { Time interval } \\
(\mathbf{m i n})\end{array}$ & \multirow{2}{*}{ BADA } & Linear & Quadratic \\
\hline \multirow{5}{*}{ LTBA-EDDF } & $360-340$ & $121,83-123,82$ & 10,00 & 9,60 & 9,63 \\
\cline { 2 - 6 } & $340-310$ & $128,4-133,32$ & 29,00 & 27,15 & 27,16 \\
\cline { 2 - 6 } & $310-80$ & $134,37-149,92$ & 103,00 & 104,60 & 104,23 \\
\cline { 2 - 6 } & $80-43$ & $151,0-155,82$ & 43,00 & 39,29 & 39,25 \\
\cline { 2 - 6 } & $43-30$ & $157,48-158,98$ & 15,00 & 13,28 & 13,32 \\
\hline \multirow{3}{*}{ KLAX-KFJK } & $370-150$ & $267,7-280,18$ & 76,87 & 72,29 & 71,95 \\
\cline { 2 - 6 } & $190-29$ & $283,37-289,87$ & 51,48 & 50,28 & 50,04 \\
\hline
\end{tabular}


For LTBA-EDDF and KLAX- KJFK flight routes, the total fuel consumption is found $193,89 \mathrm{~kg}, 193,58 \mathrm{~kg}$,
$122,57 \mathrm{~kg}$ and $121,99 \mathrm{~kg}$ for linear and quadratic models, respectively. The obtained and BADA results are presented close to each other in Table 5.

Table 5. The comparison of fuel consumption $(\mathrm{kg})$ results

\begin{tabular}{|c|c|c|c|c|c|}
\hline \multirow{2}{*}{ Flight routes } & \multicolumn{2}{|c|}{ Parameters } & \multicolumn{2}{c|}{ Fuel consumption (kg) } \\
\cline { 2 - 6 } & $\begin{array}{c}\text { Altitude } \\
(* \mathbf{1 0 0 f t})\end{array}$ & $\begin{array}{c}\text { Time interval } \\
(\mathbf{m i n})\end{array}$ & BADA & Linear & Quadratic \\
\hline LTBA-EDDF & $360-30$ & $121,83-158,98$ & 200,00 & 193,89 & 193,58 \\
\hline KLAX-KJFK & $370-29$ & $267,70-289,87$ & 128,35 & 122,57 & 121,99 \\
\hline
\end{tabular}

\section{Conclusion}

In this study, two approaches for estimating of fuel consumption are developed by using BADA. One of approaches represents the relation between altitude and fuel consumption and another indicates the dynamic change of the altitude respect to time. Using different equations, for real flight routes (LTBA-EDDF and KLAX-KJFK) two adequate fuel consumption models linear and quadratic- are constructed. For LTBA- EDDF flight routes, fuel consumption as $200 \mathrm{~kg}, 193,89 \mathrm{~kg}$ and $193,58 \mathrm{~kg}$ are found for BADA, linear and quadratic models, respectively. Furthermore, for KLAX-KJFK flight routes, $128,35 \mathrm{~kg}$ fuel consumption in terms of BADA and $122,57 \mathrm{~kg}, 121,99$ fuel consumption are computed according to linear and quadratic models, respectively. The proposed models facilitate to estimate the fuel consumption, because application of recommended models need information about dynamic of flight phases. In this paper, besides the static model used in previous studies $[1,2,4,7]$, the dynamic fuel consumption model is introduced.

In the future study, developing a fuel consumption model for different aircraft types and for the whole flight trajectory is aimed.

\section{References}

1. Senzig, D.A, Fleming, G.G, Iovinelli, R.J, Modeling of terminal area airplane fuel consumption" Journal of Aircraft, 2009, 46(4), 1089-1093.

2. Collins, B., Estimation of aircraft fuel consumption, Journal of Aircraft, 1982, 19(11), 969-975. DOI:10.2514/3.44799.

3. Trani, A., Wing-Ho, F, Schilling, G, Baik, H, and Seshadri, A, A Neural network model to estimate aircraft fuel consumption, in 4th AIAA Aviation Technology, Integration and Operations (ATIO) Forum, AIAA, Chicago, USA, 2004.

4. Baklacioglu, T, Modeling the fuel flow-rate of transport aircraft during flight phases using genetic algorithm-optimized neural networks, Aerospace Science and Technology, 2015, 49, 52-62.
5. Bartel, M., Young, T.M, Simplified thrust and fuel consumption models for modern two-shaft turbofan engines, Journal Aircraft 2008, 45 (4), 1450-1456.

6. Patterson, J., Noel, G., Senzig, D., Roof, C., Fleming, G. Analysis of departure and arrival profiles using real-time aircraft data, Journal of Aircraft, 2009, 46(4), 1094-1103.

7. Malwitz, A, Kim, B, Fleming, G., Lee, J., Balasubramanian, S., Waitz, I., Validation assessment, model assumptions and uncertainties, , SAGE, Version 1.5, FAA, 2005

8. Lee, J., Waitz I., Kim B., Fleming G., Maurice L., and Holsclaw C., System for assessing aviation's global emissions, part 2 : uncertainty assessment, Transportation Research Part D: Transport and Environment, 2007, 12(6), 381-395.

9. Hill P, Petersen C., Mechanics and thermodynamics of propulsion 2nd edn., 1992, Addison-Wesley, Reading, MA

10. Turgut, E.T, Rosen, M.A, Relationship between fuel consumption and altitude for commercial aircraft during descent: preliminary assessment with a genetic algorithm, Aerospace Science Technology, 2012, 17(1), 65-73.

11. Stolzer, A.J., Fuel consumption modeling of a transport category aircraft: a flight operations quality assurance (FOQA) analysis, Journal of Air Transportation, 2003, 8(2), 3-18.

12. Gong, C. and Chan, W.N., Using Flight Manual Data to Derive Aero-Propulsive Models for Predicting Aircraft Trajectories, AIAA Aircraft Technology, Integration and Operations Conference, 2002, Los Angeles, USA.

13. Allaire, D.L, A Physics-Based Emissions Model for Aircraft Gas Turbine Combustors, Master of Science in Aerospace Engineering at the Massachusetts Institute of Technology, 2006.

14. Spencer, K.S, Fuel Consumption Optimization Using Neura Networks and Genetic Algorithms, 2011, Dissertation for the Degree of Master of Aerospace Engineering.

15. Ryerson, M.S., Hansen M, Bonn J, Validating Aircraft Performance Models with Airline Data, International Conference on Research in Air Transportation, 2012, University of California, Berkeley.

16. Chati, Y.S, Balakrishnan, H., Aircraft Engine Performance Study Using Flight Data Recorder Archives, AIAA Aviation Conference, 2013, Los Angeles, California.

17. www.flightaware.com (accessed 10.08.2016). 